\title{
Hepatitis E Nanoparticle: A Capsid-Based Platform for Non-Invasive Vaccine Delivery and Imaging-Guided Cancer Treatment
}

\author{
Mo A Baikoghli, Chun-Chieh Chen and R Holland Cheng* \\ Department of Molecular and Cellular Biology, University of California, USA
}

Submission: February 11, 2018; Published: February 15, 2018

"Corresponding author: R Holland Cheng, Department of Molecular and Cellular Biology, University of California, Davis, CA, USA, Tel: 5309888659; Email: rhch@ucdavis.edu

\section{Abstract}

Through nanotechnology, progressively, drug delivery, cancer treatment, and vaccination are moving towards personalization. With personalized and precision medicine being one of the major focus points of nanotechnology and nanopharmacological studies these days, there is a great need for an efficient nanocarrier delivery system. Several nanocarrier systems have been introduced including both natural and synthetic material; however, overcoming biological barriers, targeting accuracy, and encapsulation efficiency remain a challenge. Here we summarize the latest breakthroughs of protein-capsid-based nanocarrier, HEVNP, as a platform for non-invasive mucosal delivery for vaccination and delivery of therapeutics.

Keywords: Protein capsid; Hepatitis e vlp; HEVNP; Nanoparticle; Cancer diagnosis; Cancer treatment; Site-specific delivery; Mucosal delivery; Non-invasive delivery

Abbreviations: VLP: Virus-Like Particles; FDA: US Federal Food and Drug Administration; HBV: Hepatitis B Virus; HEV: Hepatitis E Virus; HIV: Human Immunodeficiency Virus; NP:Nanoparticles; HPV: Human Papillomavirus; ORF2: Open Reading Frame 2; pORF: Plasmid of Open Reading Frame

\section{Introduction}

Today's medicine revolves around a "standards of care" to employ the best approach towards treatment and prevention for the general public. Meanwhile, a growing trend is aimed towards exploring precision medicine to meet the needs for each individual, accordingly. Current vaccine administration regimens by parenteral routes (i.e. intramuscular or subcutaneous injections) are poorly met with patient compliance. Use of needles is not convenient and may result in pain, allergic reactions, infections, and even nerve damage [1]. Additionally, non-specific delivery of drugs leads to suboptimal results and high toxicity [2]. Thus, major efforts are invested towards the development of therapeutic delivery via non-invasive routes to increase patient compliance and enhance targeting accuracy.

Recent developments in nanotechnology, particularly in nanodelivery systems has elevated the significance of personalized and precision medicine. Despite tremendous effort in design and development of effective drug delivery systems, overcoming biological barriers and achieving successful accumulation of nanotherapeutics at targeted site remains a challenge [3]. Thus, the primary focus in both pharmaceutical sciences and nanotechnology is to 1) enhance drug targeting and delivery, 2) reduce toxicity, 3) and achieve greater safety and biocompatibility [4].

\section{Discussion}

\section{Protein-based nanoparticles for delivery, targeting, and treatment}

Compared to conventional drug formulations, nanoparticlebased drug delivery systems offer solutions for overcoming pharmacokinetic limitations and are advantageous in prolonging the lifetime of circulating drugs $[5,6]$. Despite the success of engineered delivery vehicles, major limitations such as low encapsulation efficiency, suboptimal targeting, and undesirable degradation productions, remain a challenge [7]. A potent and effective delivery system must be equipped with following characteristics: It must be safe, biocompatible, and biodegradable; have high encapsulation efficiency and surface modification properties to enhance targeting accuracy, while 
offering protection and high retention of drug composition and bioactivity. Such delivery system must be reproducible and economically feasible.

Natural biomolecules such as protein are valuable alternatives, and even more advantageous compared to synthetic polymers or natural liposome-based drug delivery systems.
Protein-based nanocarrier systems can be prepared under mild conditions and without the use of toxic chemicals or organic solvents [8]. They are unique due to their molecular recognition, bioavailability, and biodegradability into amino acids. The natural protein building blocks, the primary amino acid sequence, offers possibilities for modifications such as covalent conjugation of drugs and targeting molecules (Figure 1).

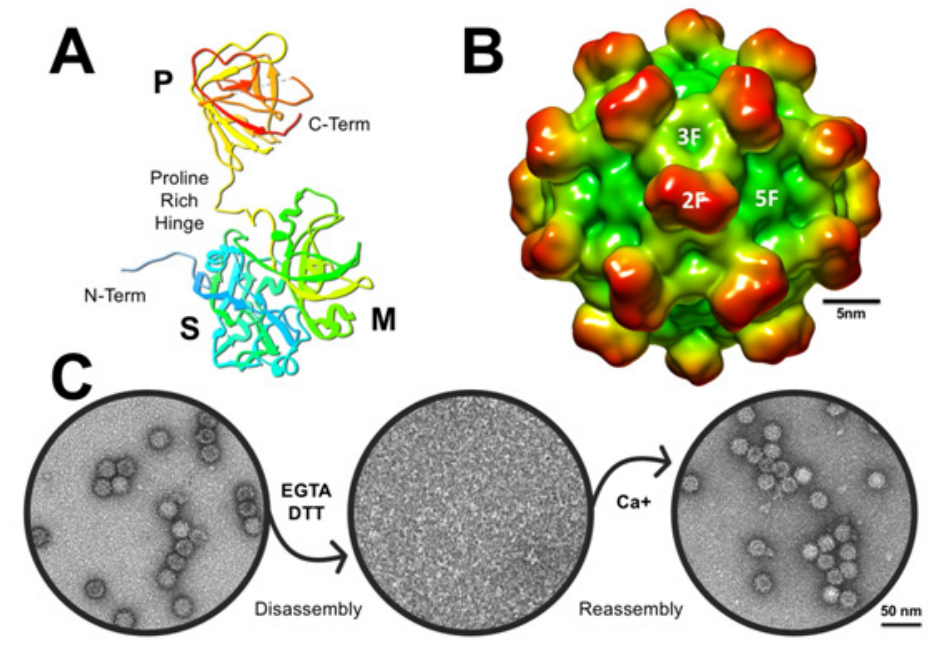

Figure 1: Hepatitis E Nanoparticle Structure, Disassembly, and Reassembly. A) HEVNP monomeric units showing the tertiary structure with $\mathrm{S}$ domain colored in blue, $\mathrm{M}$ domain in green, connected to the $\mathrm{P}$ domain yellow/red via the flexible proline rich hinge. In $\mathrm{T}=1$ configuration, 60 identical copies of monomeric HEVNP subunits are arranged in an icosahedral lattice to form the nanocapsid. B) Surface rendering of HEVNP showing the 3-Fold (3F), 2-Fold, and 5-Fold symmetry axes. Protein capsid diameter is $27 \mathrm{~nm}$. C) Process of disassembly and reassembly using EGTA (or EDTA) and DTT, and replenishment of Calcium to achieve reassembly.

Virus-Like Particles (VLP) have shown great promise in vaccination studies, as well as cancer diagnosis, targeting, and treatment [9-11]. The use of VLP-based vaccination as its own immunogen has been well-established in two FDAapproved vaccines against HBV in 1996, and HPV in 2006 [9]. In recent years, the research advancement of Hepatitis E viral nanoparticles (HEVNP) has shown great promise in encapsulation of both organic and inorganic material, as well as surface functionalization. Such genome-free capsids have been exploited as advanced drug delivery systems due to their highly symmetrical structure, bioavailability, and stability.

\section{Hepatitis E nanoparticles}

The virion-sized HEV capsid has a diameter of $\sim 45 \mathrm{~nm}$ (PDB ID: 3IYO), assembled into an icosahedral cage in an RNA dependent manner, with 180 protruding arms [12]. Genomic modifications to ORF2 plasmid (pORF2; nucleotides 5145$7125)$, include a 111 amino acid truncation at the $\mathrm{N}$-terminal and a 52 amino acid truncation at the C-terminal, which formulate an RNA independent self-assembled HEVNP with 60 protruding arms. The diameter of HEVNP is approximately $27 \mathrm{~nm}[13,14]$. The structure of HEVNP was resolved by x-ray crystallography and cryo-electron microscopy (PDB ID: 2ZZQ and 2ZTN) [15] (Figure 1).
The non-infectious and highly stable HEVNP, is comprised of three domains: S (shell domain; amino acids 118-317), M (middle domain; amino acids 318-451), and $\mathrm{P}$ (protrusion domain; amino acids 452-606) [12,16]. The $S$ domain is the most conserved regions among HEV genotypes and along with $\mathrm{M}$ domain is responsible for the formation of HEV capsid. Moreover, the $\mathrm{M}$ domain interacts strongly with the $\mathrm{P}$ domain through a long proline-rich hinge. The $\mathrm{P}$ domain serves as the primary binding site for both cellular receptor and neutralizing antibodies. 60 repeated copies of the P domain on the surface of HEVNP, provide high accessibility for surface modulations that may include imaging molecules, tracking nanoparticles, targeting ligands, and immunogenic peptides [11].

\section{Surface modulation: vaccine delivery and cancer targeting}

Recently, HIV vaccine-encapsulated HEVNP was successful in mucosal delivery and immune response in mice [17], where Jariyapong and co-workers showed that the insertion of a short 15 amino acid peptide from the V3 loop of HIV-1 gp120 (p18) into the surface of HEVNP significantly lowered detection immune system response against HEVNP [18]. Moreover, the p18HEVNP retained its icosahedral arrangement, suggesting that intermolecular forces between the recombinant nucleocapsid 
were not disrupted by the p18 insertion. The insertion was made in the antibody-binding site of the HEVNP, in the middle regions of pORF2, after the residue Tyr485. Spatial configuration of antigenic domains of HEVNP have been well characterized [19]. The chimeric HEVNP triggered an HIV-1-specific cytotoxic T-lymphocyte (CTL) response [18]. The reactivity of p18-HEVNP was tested by two antibodies, 447-52D and 224, which specifically target the V3 loop of HIV-1 gp120, and conformational epitope of WT HEV, respectively (Figure 2A).

With the success of $\mathrm{p} 18$ peptide insertion into the $\mathrm{P}$ domain of HEVNP, chemical activation of functionalized HEVNP for cancer targeting was explored as a more feasible tool for sitespecific drug delivery. In 2014 Chen and colleagues carried out site-directed mutagenesis on the $\mathrm{P}$ domain Five residues were tested: Y485, T489, S533, N573 and T586C. These sites were selected based on their 3-dimensional location as well as the feasibility of sequence mutation to minimize potential distortion of the HEVNP assembly. N573C did not disrupt HEVNP formation and showed high accessibility [20]. Subsequently, a breast cancer targeting ligand LXY30, a cyclic peptide, with selective binding to a3 integrin, was chemically conjugated to the HEVNP-573C and administered into female SPF BLAB/c mice [20,21]. Real-time in vivo fluorescence microscopy confirmed that the chemically activated HEVNP-573C-LYX30 was delivered to the breast cancer tissue successfully. HEVNP-573C-LXY30 showed higher binding to cancer cell lines as well as tumor tissue compared to WT HEVNP [11,20] (Figure 2B).

Lately, Stark et al. [22] successfully conjugated nanogold clusters (AuNC) onto HEVNP-573C nanoparticles. The study used Au102 surrounded with 44 molecules of pMBA (here after Au102pMBA44) with a of diameter of $\sim 2.5 \mathrm{~nm}$ [23]. AuNCs were directly conjugated to the exposed $573 \mathrm{C}$ through maleimide-thiol ligand exchange. Computer enhanced molecular simulations and cryo-EM single particle analysis revealed that surface conjugated Au102pMBA44-C6 nanoclusters tend to concentrate at the axial center of the 5-fold [22] (Figure 2C). Such efficient conjugation design offers insight for future ligand designs towards theranostic applications in cancer treatment.

\section{Encapsulation: delivery of nanotheranostics towards tumor treatment}

Significant effort has been invested in demonstration of HEVNP's ability to encapsulate drugs, DNA, RNA, proteins, and even inorganic beads, such as ferrite oxide nanoparticles. This is achieved by disassembling the HEVNP, adding the cargo, and reassembling the protein capsid. Removal of calcium ions is the key factor to achieve disassembly by disrupting calcium bridges. Re-supplementation of calcium to the disrupted HEVNP constituents, and removal of reducing and/or chelating agents leads to reassembly of HEVNP [12,14].

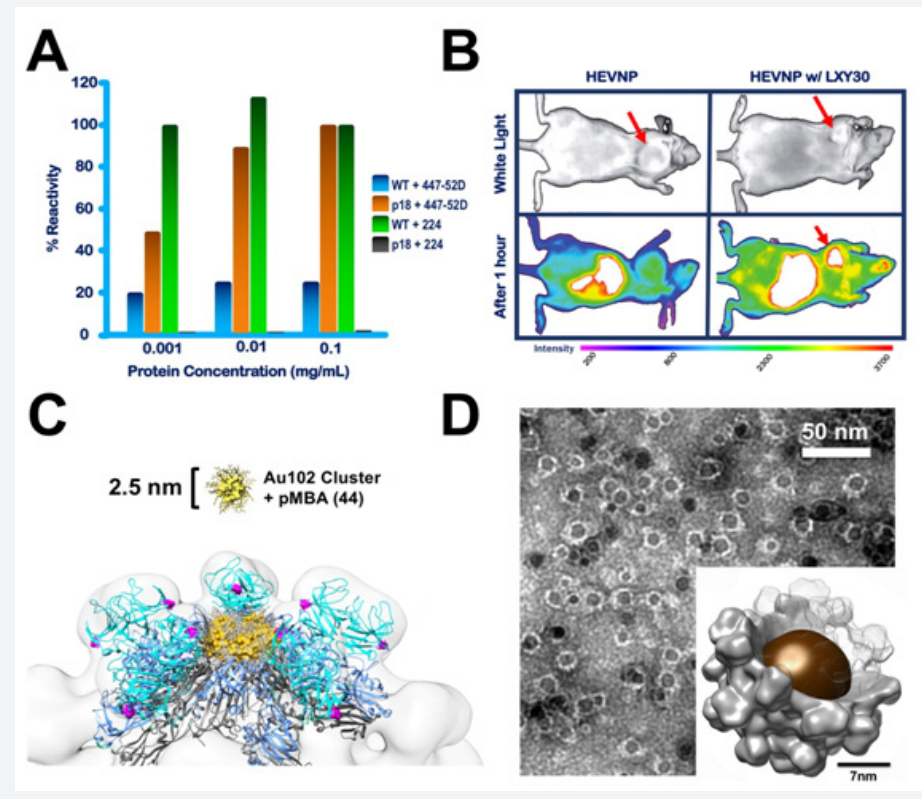

Figure 2: Recent achievements of HEVNP in vaccine delivery, tumor targeting, and encapsulation of nanotheranostics A) Reactivity of chimeric HEVNP with p18 insertion. Upon insertion of p18, HEVNP shows no reactivity to HEV-specific antibody 224. Additionally, HIV1 -specific antibody shows high reactivity to HEVNP-p18. B) In vivo imaging of breast cancer tumor targeting in mice. Without surface functionalization of HEVNP with targeting molecules, LXY30, HEVNP remain in circulation but does not accumulate at tumor site. With LXY30 conjugated to the surface of HEVNP, the nanocapsid accumulates at the tumor site. C) Surface functionalization of HEVNP with nanogold clusters, Au102. D) Encapsulation of ferrite oxide nanoparticles in HEVNP with average diameter of $30 \mathrm{~nm}$.

In 2017, Chen and colleagues illustrated successful encapsulation of ferrite oxide particles with averaged diameter of 15 nm, in HEVNP [24] (Figure 2D). This achievement is a milestone in development of tumor targeting strategies through hyperthermia treatment. Demonstration of encapsulation properties and highly modulatable surface properties make 
HEVNP an ideal candidate for vaccine delivery, tumor diagnosis, targeting, and treatment. Moreover, since Hepatitis E virus is feco-orally transmitted, by nature, its non-infectious counterpart, HEVNP is resistant to enzymatic and $\mathrm{pH}$ degradation and can be delivered at mucosally $[10,24]$.

\section{Conclusion}

HEV is a non-enveloped, feco-orally transmitted RNA virus. The genetically modified, non-infectious HEVNPs retain the natural structural stability, antigenicity, and cell binding capabilities of HEV. Biochemical engineering allows for surface modifications to functionalize the capsid-platform. The controlled disassembly and reassembly properties of HEVNP opens avenues for encapsulation of various therapeutic agents, such as DNA or RNA for gene therapy, or magnetic particles for enhancing tracking signals as well as hyperthermia cancer treatment, and proteins or peptides for metabolic disease treatment.

\section{Acknowledgement}

The authors acknowledge the sponsorship of the funding to RHC by NIH grant numbers: A1095382, EB021230, CA198880, National Institute of Food and Agriculture, as well as Finland Distinguished Professor Program.

\section{Conflict of Interest}

The authors had no conflict of interest in this review article.

\section{References}

1. Ramtahal J, Ramlakhan S, Singh K (2006) Sciatic nerve injury following intramuscular injection: a case report and review of the literature. J Neurosci Nurs 38(4): 238.

2. Cho K, Wang X, Nie S, Chen ZG, Shin DM (2008) Therapeutic nanoparticles for drug delivery in cancer. Clin Cancer Res 14(5): 13101316.

3. Blanco E, Shen H, Ferrari M (2015) Principles of nanoparticle design for overcoming biological barriers to drug delivery. Nature biotechnology 33(9): 941.

4. Torchilin VP (2005) Recent advances with liposomes as pharmaceutical carriers. Nat Rev Drug Discov 4(2): 145.

5. Torchilin V (2008) Intracellular delivery of protein and peptide therapeutics. Drug Discov Today Technol 5(2-3): e95-e103.

6. Lu Y, Sun W, Gu Z (2014) Stimuli-responsive nanomaterials for therapeutic protein delivery. J Control Release 194: 1-19.

7. Estrada LH, Champion J (2015) Protein nanoparticles for therapeutic protein delivery. Biomaterials science 3(6): 787-799.

8. Lohcharoenkal W (2014) Protein nanoparticles as drug delivery carriers for cancer therapy. BioMed research international 2014: 2014.

9. Roldão A, Mellado MC, Castilho LR, Carrondo MJ, Alves PM (2010)
Virus-like particles in vaccine development. Expert Rev Vaccines 9(10): $1149-1176$

10. Holla P (2017) Toward Mucosal DNA Delivery: Structural Modularity in Vaccine Platform Design, in Micro and Nanotechnology in Vaccine Development, pp. 303-326.

11. Stark M, Cheng RH (2016) Surface modulatable nanocapsids for targeting and tracking toward nanotheranostic delivery. Pharm Pat Anal 5(5): 307-317.

12. Xing L, Li TC, Mayazaki N, Simon MN, Wall JS, et al. (2010) Structure of hepatitis $\mathrm{E}$ virion-sized particle reveals an RNA-dependent viral assembly pathway. Journal of Biological Chemistry 285(43): 3317533183.

13. Li TC, Takeda N, Miyamura T, Matsuura Y, Wang JC, et al. (2005) Essential elements of the capsid protein for self-assembly into empty virus-like particles of hepatitis E virus. J Virol 79(20): 12999-13006.

14. Xing, L (1999) Recombinant hepatitis E capsid protein self-assembles into a dual-domain $\mathrm{T}=1$ particle presenting native virus epitopes. Virology 265(1): 35-45.

15. Wang CY, Miyazaki N, Yamashita T, Higashiura A, Nakagawa A, et al. (2008) Crystallization and preliminary X-ray diffraction analysis of recombinant hepatitis E virus-like particle. Acta Crystallogr Sect F Struct Biol Cryst Commun 64(4): 318-322.

16. Yamashita T, Mori Y, Miyazaki N, Cheng RH, Yoshimura M, et al. (2009) Biological and immunological characteristics of hepatitis $\mathrm{E}$ viruslike particles based on the crystal structure. Proc Natl Acad Sci U S A 106(31): 12986-12991.

17. Takamura S, Niikura M, Li TC, Takeda N, Kusagawa S, et al. (2004) DNA vaccine-encapsulated virus-like particles derived from an orally transmissible virus stimulate mucosal and systemic immune responses by oral administration. Gene Ther 11(7): 628-635.

18. Jariyapong P, Xing L, van Houten NE, Li TC, Weerachatyanukul W, et al. (2013) Chimeric hepatitis E virus-like particle as a carrier for oraldelivery. Vaccine 31(2): 417-424.

19. Xing L, Wang JC, Li TC, Yasutomi Y, Lara J, et al. (2011) Spatial configuration of hepatitis E virus antigenic domain. J Virol 85(2): 11171124.

20. Chen CC, Xing L, Stark M, Ou T, Holla P, etal. (2016) Chemically activatable viral capsid functionalized for cancer targeting. Nanomedicine 11(4): 377-390.

21. Xiao W, Li T, Bononi FC, Lac D, Kekessie IA, et al. (2016) Discovery and characterization of a high-affinity and high-specificity peptide ligand LXY30 for in vivo targeting of $\alpha 3$ integrin-expressing human tumors. EJNMMI research 6(1): 18.

22. Stark MC (2017) Structural characterization of site-modified nanocapsid with monodispersed gold clusters. Scientific reports 7(1): 17048.

23. Levi-Kalisman (2011) Synthesis and Characterization of Au102 (p-MBA) 44 Nanoparticles. Journal of the American Chemical Society 133(9): 2976-2982.

24. Chun-Chieh Chen, Marie Stark, Mo A Baikoghli, R Holland Cheng, et al. (2017) Hepatitis E Virus Nanoparticle Encapsulating Nano-Theranostic Reagent as Modularized Capsule. Juniper Publisher, Advanced Research in Gastroenterology \& Hepatology 5(5): 555674. 


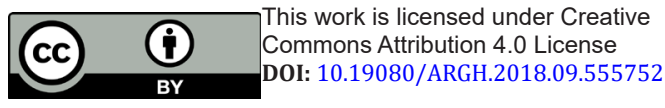

Your next submission with JuniperPublishers will reach you the below assets

- Quality Editorial service

- Swift Peer Review

- Reprints availability

- E-prints Service

- Manuscript Podcast for convenient understanding

- Global attainment for your research

- Manuscript accessibility in different formats

( Pdf, E-pub, Full Text, audio)

- Unceasing customer service

Track the below URL for one-step submission https://juniperpublishers.com/online-submission.php 